\title{
Applications of Extended Reality Systems in Aesthetic Medicine Training
}

\section{Saad AlSogair*}

Elite Derma Care Clinic, Dr. Layla Al-Onaizi Polyclinic, Khobar, Saudi Arabia

*Corresponding Author: Saad AlSogair, Elite Derma Care Clinic, Dr. Layla Al-Onaizi

Polyclinic, Khobar, Saudi Arabia.
Received: June 16, 2021

Published: July 23, 2021

(C) All rights are reserved by Saad AlSogair.

\begin{abstract}
Extended reality (XR) systems provide a modernistic approach to training specialists in high-risk professions. Virtual reality (VR) and augmented reality (AR) are starting to get utilized more frequently in medical training, preoperative planning, and rehabilitation. With the increased popularity of aesthetic procedures, the demand for a more modernistic approach to training specialists is also growing. Furthermore, the Covid-19 pandemic has shown us the importance of digital education, and improvements in online teaching will be necessary in the future. However, XR simulations are not completely translatable to real experiences and further research is needed for developing substitutes of live and cadaver models. In this article, we provide a review of the XR systems, developed for aesthetic medicine training and their applicability, compared to traditional methods.
\end{abstract}

Keywords: Extended Reality; Virtual Reality; Augmented Reality

\section{Introduction}

The demand for cosmetic interventions has increased exponentially in the last decade. A 2019 statistical report of "The American Society of Plastic Surgeons" shows up to a 5318\% increase in some cosmetic surgical procedures since 2000 , and $878 \%$ for some nonsurgical minimally-invasive procedures in the US [1].

Aesthetic medicine combines practices used for improving cosmetic appearance through both surgical and non-surgical procedures, such as the removal of skin imperfections or unwanted hair, insertion of dermal fillers and implants, and reconstructive surgery. Aesthetic medicine practices have become more abundant, which leads to the necessity of more specialists and more efficient training mechanisms. With the increased number of procedures, serious procedure-related complications also rise. Training of greater effectiveness is needed, due to limitations of the traditional methods. Usual methods include practice on living volunteer pa- tients and animals or cadavers, which raises some ethical, legal, and safety concerns. Augmented reality (AR) and virtual reality (VR) simulations are one modern approach to this problem [2].

VR uses a computer device to simulate an interactive experience that can be similar to the real world or to introduce completelyaltered reality. VR systems use special headsets or multi-projected rooms to stimulate the senses and change the surroundings of the user. Furthermore, haptic devices can be utilized to reproduce the sense of touch by exerting forces, vibrations, or motions. AR is an interactive experience, which only adds digital elements to a real landscape, and extended reality (XR) is a generalized term that covers all of the technologies that enhance the senses and experience of the user, including VR and AR.

VR was used for the first time in healthcare at the beginning of the 1990s for visualization of medical data during surgery and preoperative planning of surgical procedures [3]. Since then, AR and 
VR are being utilized in various medical fields for planning and practicing different medical manipulations and their applicability in surgical training has been well-documented in the literature for a wide spectrum of different surgical subspecialties [4]. This includes plastic surgery and other cosmetic procedures, such as filler injection, which is one of the most commonly performed aesthetic practices [5]. The overall low cost and efficiency of these training methods have given rise to research and innovations.

This article aims to discuss the application of XR training systems in the aesthetic medicine field.

\section{Methodology}

A literature search was made through PubMed and Google Scholar, utilizing the following keywords "virtual reality", "VR", "augmented reality", "AR", "aesthetic medicine", "plastic surgery", "aesthetic surgery", "dermatology", "training", "education". Fortythree articles were selected for review. Abstracts were scanned for relevance and a total of thirty articles were observed for the current study. Bibliographies were checked for relevant sources, and headlines, related to the topic were also inspected.

\section{Results and Discussion}

AR and VR are showing great potential in the simulation of high-risk tasks and are becoming a preferred training method for such professions [6,7]. Continuous repetition of hazardous tasks in a controlled environment can be greatly beneficial for building the necessary reflexes and confidence. On the other hand, such systems can be very useful for the assessment of trainees, due to the ability to fragment complex tasks into simple assignments and evaluate the score, achieved by the student [8]. The utilization of XR in the medical field is expected to increase five times by 2025 . Systems of such type can optimize preoperative planning with digital visualization of the expected income, derive easier access to information in the operating room, and potentially improve rehabilitation [9]. They can be a revolutionary approach for training surgical and diagnostic skills and an easier and more fascinating method for learning medicine [10].

\section{Implant placement}

Seung Min Oh., et al. reported about the use of a VR system in filler injection training. The technique for injecting fillers is a blind technique, which predisposes to vessel damage or even intravascular injection. This can lead to serious complications, including blindness and stroke.
A VR system was developed by Maniamind Co., Ltd. in Seoul, Republic of Korea. A human face 3D model was designed by using the data from cadaveric examination, computer tomography, and thin-slice (1mm interval) magnetic resonance imaging. Skin and skull emplacement was defined by examining the cadaveric CT scan data. Soft tissue data was extracted from the live-body thin-slice MRI scan and live-body CT scan. The VR-based filler training system was examined by a total of 100 physicians. Half of them were aged between 35 and 50 and 38\% had more than 5 years of experience in aesthetics. Participants, aged mostly under 35 rated the system as having good usability, and the ones that were more likely to rate the system as having poor usability were aged between 35 and 50. This indicates that the system is more applicable amongst trainees, rather than specialists. In total, three-fourths of the participants rated the system as having 'acceptable' usability, stating that it increases anatomical understanding of vessels and nerves and raises awareness for a safer procedure [5].

\section{Reconstructive surgery}

Fang., et al. observed a haptics-based VR temporal bone simulator for anatomy and surgery training. The system allowed users to adjust the transparency and opacity of the key structures and explore the sinus, middle and posterior fossa, inner ear, and facial nerve. The haptic device represented a drill, which allowed users to adjust pressure and feel the resistance of different tissue. There was a pop-out notification when the user was approaching vital structures.

7 otolaryngology residents and 7 medical students participated in the examination of this simulator. Questionnaires showed that residents rated cadaver temporal bone dissection training as more functional than training with the simulator. The residents agreed that a plastic temporal bone was better in sense of reality, but the VR system was more successful as a tool for anatomy learning. In general, residents were satisfied with the system's efficacy in improving performance. All medical students stated that they have improved cognition in anatomic structures, but needed more simulation sessions for achieving complete proficiency [11].

Microsurgery is a technique that requires an extremely high level of precision and dexterity. This can be achieved only by continuous repetition of certain techniques. Cadavers and live animals are traditional training models, but are also exhaustible consumables and raising some ethical concerns. That is why an appropriate XR system can be very useful for training specialists, as such simula- 
tion can be repeated infinite times with lower overall cost. Currently, there is a variety of commercial suturing technique simulators, that one can choose from [12].

Kazemi., et al. developed a virtual-reality-based system for microsurgical trainees and assessed its accessibility and functionality through four groups of individuals with different levels of medical experience. Participants included microvascular surgeons with an average experience of 7 years, medical students who had gone through microsurgical training, and two groups of non-specialists. All participants performed suturing techniques using the simulator.

The VR system allowed assessment of stylus oscillations, speed and quality of work, tissue trauma frequency and damage in the areas, penetration and exit angles, frequency of needle orientation changes, and effect of needle shape in performance. According to oscillation, younger groups had better results, showing less tremors compared to microsurgery specialists. The four groups showed no significant differences in performance, quality, and speed, although medical students and surgeons caused less tissue trauma than non-specialists [12].

VOXEL-MAN TempoSurg ${ }^{\circledR}$ is a commercially available VR simulator designed for preoperative planning of temporal bone surgery and teaching trainees. The product was designed, using 3D models of the skull, derived from CT scan data, and is equipped with a haptic device, allowing the user to control a virtual drill and get feedback on the resistance of different structures [13].

Stephen Schendel and colleagues reported about the development of a computer-based simulation system for preoperative planning and training of cleft-lip repair. The system was equipped with a haptic force-feedback device, which represented a virtual cutting tool and forceps. The model of the patient was derived from the CT scan of an 11-day-old patient with cleft lip and palate deformation. The simulation system aimed to achieve repetitive training and assessment of the preoperative planning of the user, prompting the selection of anatomical landmarks, which are then compared against that of a gold standard. A score is then generated, according to the deviation from the standard and the required time. The system was tested on plastic surgery residents and people without medical background, and the residents scored notably better. The score of all participants increased with practice and the time needed to complete the task decreased [14].
Oliker., et al. used 3D virtual model software, created by BioDigital Systems, to train and assess the surgical knowledge of residents. 3D models were created to fit four surgical procedures in a VR environment: Monobloc, Le Fort III, Fronto-orbital advancement, and Latissimus flap breast reconstruction. The models were designed using patient-specific CT scan and MRI [15].

Khelemsky., et al. investigated the Touch Surgery ${ }^{\mathrm{TM}}$ application, designed for teaching and assessment of surgical skills. The study was aimed towards orbital floor reconstruction (OFR). To investigate the utility of this simulator, the authors of the study, invited novice dental graduate students and OFR surgery experts to test their knowledge on the simulator. Both groups rated the simulation as favorable in means of anatomic accuracy, surgical instrumentation, and surgical sequence, but experts' appreciation was slightly lower than the novices. Currently, 43 other plastic, reconstructive and aesthetic surgery procedure simulations are available for training in the application [16].

\section{D face models}

In 2005, Smith., et al. introduced a 3D computer model, which illustrated the human face and allowed observation of the aging process. The VR model was also used to demonstrate finger-assisted malar elevation and superficial musculoaponeurotic system (SMAS) manipulation in rhytidectomy. The authors aimed to make a tool for surgical training and improve preoperative planning. The system allowed users to isolate distinct structures and observe the model, while the rest of the face ages, thus allowing visualization of the aging process and how it changes the architecture of the face [17].

Kumar., et al. reported about the development of a virtual 3D model of the human face from a real cadaver, using a photogrammetry technique. The model had only one hemiface dissected and the other was left intact, aiming for better observation of the different structures, by comparative relation. The authors asked 12 specialists, with experience between 12 and 18 years, to validate the accuracy and functionality of the simulator. Participants suggested the addition of a haptic device, more anatomical variations, and more vascularity details. Their overall appreciation of the product was 4,5 from 5 [18].

Smith and his colleagues, was also the first to develop a virtual reality atlas of craniofacial anatomy. The 3D model of the skull included bone and muscle structures, nerves and blood vessels, me- 
dial canthal apparatus, and elements of the lacrimal system. The user could adjust the opacity and the view angle of different structures and isolate them from the whole. Lingula, ante-lingula, infraorbital foramen, and anterior and posterior ethmoidal foramina were also included in the atlas [19].

Heppt., et al. created an interactive 3D model of the nose. A 3D computer-aided design (CAD) model of the cartilage and bone structures was composed and a 3D CAD transparent layer of skin was added. This design was created to improve preoperative planning and serve as a tool for medical training. Three functions were available. The first allowed observation of distinct structures of the nose or the whole nasal framework, with optional adjustment of zoom and rotation. The second function enabled alteration of the nasal framework and visualization of the procedure's possible outcome, and the third option allowed observation of commonly performed procedures. The presented 3D model allowed better preoperative planning and presentation of the possible outcome to the patient, graphic documentation of operative measures, and easier learning for medical students [20].

\section{Dermatology}

$\mathrm{AR}$ and VR have the potential to be greatly advantageous for dermatology education, as visual assessment is crucial for accurate diagnostics in this field [21].

Noll., et al. investigated the mARble ${ }^{\circledR}$, an AR-based application for Apple's iOS. This program was made for training dermatology students to distinguish different skin anomalies and can be used in a classroom environment or for self-training. The app uses paper marks with distinct patterns, which represent different skin abnormalities. Once placed on the skin, the paper marks can be seen through the screen of a smart device as the pathology they represent. Studies show that teaching with this application shows increased learning efficiency, compared to textbook learning, and also higher fascination amongst students [10].

\section{Conclusion}

Technologies have evolved greatly in the 21st century and XR systems are being utilized more frequently at training specialists, especially when it comes to high-responsibility professions, such as aviation and surgery.

Also, in the last year, digital education is being increasingly appreciated, due to the SARS-CoV-2 pandemic. However, this ap- proach is still gradually entering the medical field since learning medicine is a very complex process and is bonded to a lot of doctorpatient interactions. AR and VR utilization is increasing in aesthetic medicine and being applied more often as part of preoperative planning and education programs. Yet the classical training methods are crucial for achieving the dexterity and precision, needed for performing a successful medical procedure, due to the absence of high-quality haptic devices. Further research is needed for developing XR systems, that are completely translatable to the operating table and can eliminate cadaver and live models.

\section{Bibliography}

1. American society of plastic surgeons. "Plastic Surgery Statistics Report" (2019).

2. Hammoud MM., et al. "To the point: medical education review of the role of simulators in surgical training". American Journal of Obstetrics and Gynecology 199.4 (2008): 338-343.

3. Chinnock C. "Virtual reality in surgery and medicine". Hospital Technology Ser. 13.18 (1994): 1-48.

4. Papanikolaou IG., et al. "Changing the way we train surgeons in the 21th century: A narrative comparative review focused on box trainers and virtual reality simulators". European Journal of Obstetrics and Gynecology and Reproductive Biology 235 (2019): 13-18.

5. Oh SM., et al. "Development and Usability of a Virtual RealityBased Filler Injection Training System". Aesthetic Plastic Surgery 44 (2020): 1833-1842.

6. Van Wyk E and de Villiers R. "Virtual reality training applications for the mining industry". Proceedings of the 6th International Conference on Computer Graphics, Virtual Reality, Visualisation and Interaction in Africa - AFRIGRAPH '09 (2009).

7. Ka-Chun Siu., et al. "Adaptive Virtual Reality Training to Optimize Military Medical Skills Acquisition and Retention". Military Medicine 181.5 (2016): 214-220.

8. Abi-Rafeh J., et al. "Nonbiological Microsurgery Simulators in Plastic Surgery Training". Plastic and Reconstructive Surgery 144.3 (2019): 496e-507e.

9. CWSMSMASTD Bellini H. "Profiles in Innovation". Virtual and Augmented Reality. Understanding the Race for the Next Computing Platform. Goldman (2016).

10. Noll C., et al. "Demo". Proceedings of the 2014 Workshop on Mobile Augmented Reality and Robotic Technology-Based Systems - MARS '14 (2014). 
11. Fang TY., et al. "Evaluation of a haptics-based virtual reality temporal bone simulator for anatomy and surgery training". Computer Methods and Programs in Biomedicine 113.2 (2014): 674-681.

12. Kazemi H., et al. "Assessing suturing techniques using a virtual reality surgical simulator". Microsurgery 30.6 (2010): 479-486.

13. Linke R., et al. "Assessment of skills using a virtual reality temporal bone surgery simulator". Acta otorhinolaryngologica Italica: Organo Ufficiale Della Societa Italiana Di Otorinolaringologia e Chirurgia Cervico-Facciale 33.4 (2013): 273-281.

14. Schendel S., et al. "A surgical simulator for planning and performing repair of cleft lips". Journal of Craniomaxillofacial Surgery 33.4 (2005): 223-228.

15. Oliker A., et al. "Step-based cognitive virtual surgery simulation: an innovative approach to surgical education". Studies in Health Technology and Informatics 173 (2012): 325-327.

16. Khelemsky R., et al. "Validation of a Novel Cognitive Simulator for Orbital Floor Reconstruction". Journal of Oral and Maxillofacial Surgery 75.4 (2017): 775-785.

17. Smith DM., et al. "Applications of Virtual Reality in Aesthetic Surgery". Plastic and Reconstructive Surgery 116.3 (2005): 898-904.

18. Kumar N., et al. "A Novel Three-Dimensional Interactive Virtual Face to Facilitate Facial Anatomy Teaching Using Microsoft HoloLens". Aesthetic Plastic Surgery (2021).

19. Smith DM., et al. "A Virtual Reality Atlas of Craniofacial Anatomy". Plastic and Reconstructive Surgery 120.6 (2007): 16411646.

20. Heppt WJ., et al. "An interactive three-dimensional nose model for rhinosurgery". Facial Plastic Surgery 29.2 (2013): 121-126.

21. Sharma P., et al. "Augmented Reality in Dermatology: AR we ready for it?" Journal of the American Academy of Dermatology 81 (2019): 1216-1222.

Volume 4 Issue 8 August 2021

(C) All rights are reserved by Saad AlSogair. 